\title{
Scanning transmission X-ray microscopy with efficient X-ray fluorescence detection (STXM-XRF) for biomedical applications in the soft and tender energy range
}

\author{
Lars Lühl, Konstantin Andrianov, Hanna Dierks, Andreas Haidl, Aurelie \\ Dehlinger, Markus Heine, Jörg Heeren, Thomas Nisius, Thomas Wilhein \\ and Birgit Kanngießer
}

J. Synchrotron Rad. (2019). 26, 430-438

\section{IUT IUCr Journals CRYSTALLOGRAPHY JOURNALS ONLINE \\ Copyright (C) International Union of Crystallography \\ Author(s) of this article may load this reprint on their own web site or institutional repository provided that this cover page is retained. Republication of this article or its storage in electronic databases other than as specified above is not permitted without prior permission in writing from the IUCr. \\ For further information see http://journals.iucr.org/services/authorrights.html}




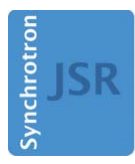

JOURNAL OF SYNCHROTRON RADIATION

ISSN $1600-5775$

Received 7 August 2018

Accepted 27 November 2018

Edited by S. M. Heald, Argonne National Laboratory, USA

Keywords: X-ray microscopy; X-ray fluorescence; biomedical applications.

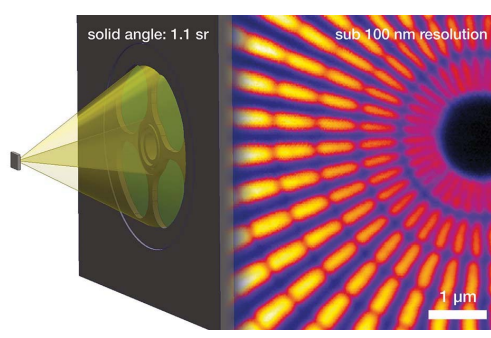

C 2019 International Union of Crystallography

\section{Scanning transmission X-ray microscopy with efficient X-ray fluorescence detection (STXM-XRF) for biomedical applications in the soft and tender energy range}

\author{
Lars Lühl, ${ }^{a} *$ Konstantin Andrianov, ${ }^{\text {b }}$ Hanna Dierks, ${ }^{a}$ Andreas Haidl, ${ }^{\text {b }}$ \\ Aurelie Dehlinger, ${ }^{a}$ Markus Heine, ${ }^{c}$ Jörg Heeren, ${ }^{c}$ Thomas Nisius, \\ Thomas Wilhein ${ }^{\mathrm{b}}$ and Birgit Kanngießer ${ }^{\mathrm{a}}$
}

\footnotetext{
anstitute of Optics and Atomic Physics, Technische Universität Berlin, Hardenbergstraße 36, Berlin 10623, Germany, bInstitute for X-Optics, University of Applied Sciences Koblenz, Joseph-Rovan-Allee 2, Remagen 53424, Germany, and 'Institute of Biochemistry and Molecular Cell Biology, UKE Hamburg-Eppendorf, Martinistraße 52, Hamburg 20246, Germany. *Correspondence e-mail: lars.luehl@physik.tu-berlin.de
}

Scanning transmission X-ray microscopy, especially in combination with X-ray fluorescence detection (STXM-XRF) in the soft X-ray energy range, is becoming an increasingly important tool for life sciences. Using X-ray fluorescence detection, the study of biochemical mechanisms becomes accessible. As biological matrices generally have a low fluorescence yield and thus a low fluorescence signal, high detector efficiency (e.g. large solid angle) is indispensable for avoiding long measurement times and radiation damage. Here, the new AnImaX STXM-XRF microscope equipped with a large solid angle of detection enabling fast scans and the first proof-of-principle measurements on biomedical samples are described. In addition, characterization measurements for future quantitative elemental imaging are presented.

\section{Introduction}

X-ray microscopy (XRM) can be performed either in full-field (FF-XRM) or scanning mode (STXM) (Eichert, 2015; Lider, 2017). In full-field mode the whole field of view is illuminated at once, whereas in scanning mode the sample is scanned by a focused beam and the signal for each point is recorded by an area detector. This makes STXM a more time-consuming method than FF-XRM, but has the advantage that a larger variety of contrast modes, such as absorption contrast, differential phase contrast and dark field contrast, can be performed simultaneously (Hornberger et al., 2008). Furthermore, the small focus of the incoming beam on the sample allows the fluorescence radiation emitted by the excited atoms in the sample to be associated with a confined spatial position. The emitted fluorescence signal can be collected by a silicondrift detector (SDD), so that the elemental composition of the sample can be determined. One of the unique features of the STXM-XRF setup presented here is the use of a four-channel SDD with flat design enabling a very large solid angle of detection (see Figs. 1 and 2).

FF-XRM, on the other hand, is not suitable for fluorescence microscopy, whereas phase retrieval is feasible but would require the implementation of additional optical elements such as Zernicke optics (Holt et al., 2013). This is a complex task, since these optics (also known as phase rings) must be adapted to every sample, and the alignment as well as the 
phase matching can be challenging. While FF-XRM can be performed at both synchrotron facilities and in the laboratory, scanning XRM depends on high-excitation intensities to avoid very long measuring times and is therefore available at synchrotron facilities only.

Synchrotron facilities at which FF-XRM in the soft and tender energy range has been established are: ALBA, ALS, Astrid, BESSY, Elettra, NSRL and Ritsumeikan. STXM measurements can be performed at ALS, BESSY, CLS, Diamond, Elettra, Photon Factory, SLS, SSRF and SSRL (Obst \& Schmid, 2014). STXM in combination with fluorescence detection (STXM-XRF) can be found at CLS, Diamond and Elettra (TwinMic). CLS and Diamond use conventional SDDs with rather small angles of detection for the fluorescence signal. The TwinMic setup at Elettra includes a detector system of eight conventional SDDs arranged circularly around the sample. A new system with a larger solid angle of detection is also under development and is planned to be operational soon (Gianoncelli et al., 2016; Bufon et al., 2018).

The soft and tender energy range is particularly well suited for applications in life sciences, as it provides a higher sensitivity for the lighter elements. Due to the low fluorescence when investigating biological matrices, a high detector efficiency (achievable through a large solid angle) is indispensable for avoiding long measurement times and radiation damage.

Our novel AnImaX (analytical imaging with X-rays) endstation, that has been tested at the high-brilliance beamline P04 at PETRA III (DESY, Hamburg), features an integrated four-channel fluorescence detector with a large solid angle of detection of up to 1.1 sterad and can therefore combine STXM with fluorescence microscopy. The AnImaX microscope is designed as a flexible endstation adaptable to various beamlines. Alignment and first resolution tests with test patterns can be carried out within a day and a quick change between FF-XRM and STXM-XRF is possible [similar to TwinMic at Elettra (Kaulich et al., 2003)]. However, the focus of this work will be on the characteristics and applications of the STXM-XRF mode.

In order to develop the fluorescence microscopy mode towards analytical imaging, i.e. towards the quantitative evaluation of fluorescence signals, the limit of detection (LOD) for some selected elements has been determined through the measurement of thin-layered samples (Hönicke et al., 2018). These samples, with layer thicknesses in the nanometre regime, were specifically designed and produced for this purpose and possess a very homogeneous lateral distribution.

The first proof-of-principle experiments with respect to fluorescence microscopy were carried out to determine the iron distribution in brown adipose tissue (BAT). In response to cold exposure, BAT generates heat by a process called adaptive thermogenesis to maintain body temperature in cold environments (Heeren \& Scheja, 2018). Accordingly, BAT has a high demand of energy-rich fuels and we used BAT activation to visualize the uptake of lipoproteins that were labelled with superparamagnetic iron oxide nanoparticles (Bruns et al., 2009).
As radiation damage is often a serious issue for biological samples, we will provide an estimation of the radiation dose in different biomedically relevant matrices. In combination with our experimental results, these considerations demonstrate the necessity of a cryogenic environment for the samples.

\section{Scanning transmission X-ray microscopy}

Due to its flexible design, the presented scanning transmission $\mathrm{X}$-ray microscope with X-ray fluorescence detection (STXM$\mathrm{XRF}$ ) is compatible with different beamlines. Since the resolution of a microscope in scanning mode depends on the size of the illumination spot, it is important to achieve a small $\mathrm{X}$-ray focus on the sample. For this, our STXM-XRF is equipped with a zone plate (ZP) optic in combination with a central stop and an order-sorting aperture (OSA) (Fig. 1). For each scanning point, the transmitted signal is captured by a CCD area detector, so that additional contrast modes such as differential phase contrast can be exploited.

Furthermore, a four-channel SDD detector has been implemented into the setup so that simultaneous XRF measurements can be performed by recording the fluorescence emission spectrum for each scanning point. The detector consists of four independent SDDs aligned around a central hole through which the excitation beam is focused onto the sample. This geometry represents a unique feature of the AnImaX endstation, as it results in a very small distance between the detector and the sample, inducing a large angle of detection (Fig. 2).

\subsection{AnImaX endstation}

The AnImaX endstation is flexible in two ways. Firstly, it allows a quick change between full-field transmission

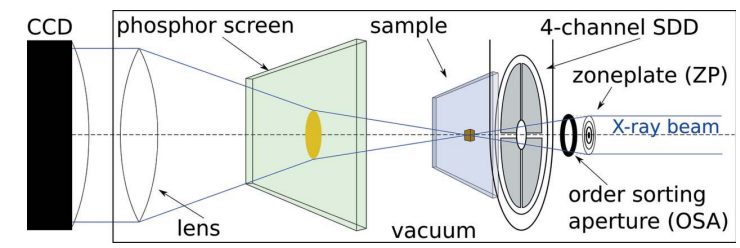

Figure 1

Sketch of the STXM setup.

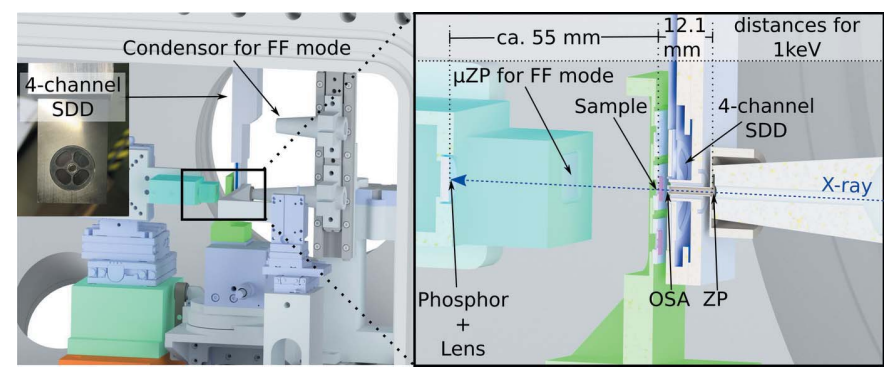

Figure 2

Drawing of the STXM setup with four-channel SDD entering from the top. For $1 \mathrm{keV}$, the distances of the phosphor, sample and $\mathrm{ZP}$ to each other are given. 
(FF-XRM) and STXM-XRF mode. Secondly, it can easily be adapted, connected and transported to various beamlines without the need for special equipment. For experienced users, connecting the endstation to the beamline and aligning the STXM-XRF to the beam can be carried out within approximately $12 \mathrm{~h}$.

In Fig. 1 an outline of the experimental setup depicts the arrangement of the optical elements. Fig. 2 shows a detailed drawing of the setup inside the vacuum chamber together with important distances for an excitation energy of $1 \mathrm{keV}$. The beam is focused by a Fresnel ZP with an outer diameter of $333 \mu \mathrm{m}$, an outer-zone width of $45 \mathrm{~nm}$ and a central stop of $160 \mu \mathrm{m}$ in diameter. At a distance of approximately two-thirds of the focal length, a circular pinhole with a diameter of $150 \mu \mathrm{m}$ serves as the OSA to suppress unwanted diffraction orders. The four-channel SDD is mounted from the top and is provided with a manual manipulator in three dimensions. This allows us to precisely align the four-channel SDD (XFlash 5060F from Bruker Nano $\mathrm{GmbH}$ ) with the optical axis (manipulator in the $x$ and $y$ directions) and adjust its distance to the sample (manipulator in the $z$ direction). The manipulator provides a large setting range, so that the four-channel SDD can be fully removed from the optical alignment by using the $y$ manipulator (up-down).

The sample is mounted on a piezo scanner with a theoretical positioning accuracy of $0.1 \mathrm{~nm}$ and a total range of $100 \mu \mathrm{m} \times$ $100 \mu \mathrm{m}$. The scanner itself is mounted on a coarse positioning stage that can be moved by piezos in the range of $63 \mathrm{~mm}$ in the $x$ direction (horizontal to the optical axis) and $31 \mathrm{~mm}$ in the $y$ direction (vertical to the optical axis) with an accuracy of $3 \mathrm{~nm}$. Up to eight samples can be placed onto the sample holder, allowing the user to quickly switch between samples.

Placed behind the sample, a phosphor screen (P43) is excited by the transmitted X-rays and emits visible light. Due to the combination of the zone plate central stop and the OSA, the illumination has the shape of a hollow cone, resulting in a characteristic ring-shaped intensity distribution on the phosphor screen. The visible radiation emitted by the phosphor screen is collected by an optical achromatic lens (12.5 mm diameter, $f=15 \mathrm{~mm}$ ) and detected by a fast-readout CCD camera (Andor IXon3 860, $128 \times 128$ active pixels with $24 \mu \mathrm{m} \times 24 \mu \mathrm{m})$ located outside the vacuum chamber equipped with an objective (Olympus, $f=75 \mathrm{~mm}-150 \mathrm{~mm}$, $\mathrm{F} / 4.0$ ). Despite the low detection efficiency of this optical system, it is the acquisition time of the four-channel SDD that limits the total scanning time.

For STXM-XRF mapping, the sample is scanned in a plane perpendicular to the optical axis. This can be carried out either step-by-step, where the sample position is fixed for each measurement, or on-the-fly, for which the sample continuously moves during the measurements. On-the-fly scans can be controlled based on either time or position. For timecontrolled measurements, the scanning positions and step widths are calculated based on acquisition time and velocity, whereas position-controlled measurements require encoder signals from the scanner, so that each measurement can be started when the sample has reached a certain position. The results presented in this paper have been acquired in timecontrolled mode.

One of the main challenges for successful STXM-XRF measurements is the proper synchronization of the scanner and the detectors for both the transmission and fluorescence measurements. Small mismatches of the scanner-to-detector synchronization might render time-consuming measurements unusable. Furthermore, it is necessary to synchronize the detectors with one another in order to compare the morphology of a sample (obtained by transmission measurements) with the elemental distribution in the sample (obtained by fluorescence detection).

The time-controlled scans in the presented STXM-XRF are based on transistor-transistor logic (TTL). At the start of each line, the scanner sends a signal to the transmission CCD. The CCD then collects a defined number of images with a defined acquisition time and waits for the next signal. For the acquisition time of the CCD, a constant TTL signal for the SDD is provided. Time delays are in the range of $<10 \mu$ s due to the electric connections $(\sim \mathrm{ns})$ and response times of the CCD $(2-6 \mu \mathrm{s})$ and the SDD $(\sim 1-2 \mu \mathrm{s})$.

As shown in Fig. 3, a time-controlled on-the-fly scan of a Siemens star test pattern (smallest structures: $50 \mathrm{~nm}$ ) was performed without any artefacts, which indicates that the synchronization of scanner and CCD operates correctly. The Siemens star consists of a $\sim 150 \mathrm{~nm}$ thick $\mathrm{W}$ layer on top of a $100 \mathrm{~nm} \mathrm{Si}_{3} \mathrm{~N}_{4}$ window. Fig. 3 shows an image recorded with an excitation energy of $720 \mathrm{eV}$ in transmission mode only. The synchronization of the CCD detector with the four-channel SDD was tested for several other samples, e.g. for biomedical applications, which will be discussed below.

Since a Siemens star pattern displays a continuous range of structure sizes, i.e. spatial frequencies, it is a well suited test object to determine not only the spatial resolution limit of a microscope but also its contrast transfer function (CTF). In terms of the frequency spectrum of the object, the imaging system can be considered as a system that tranfers the spatial frequency from the object to the image plane. The Siemens

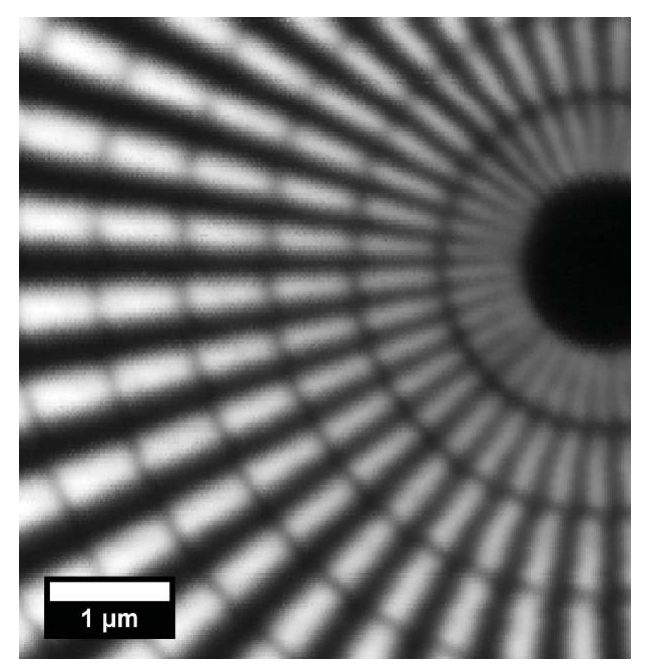

Figure 3

Transmission signal of a test pattern acquired in on-the-fly mode. 


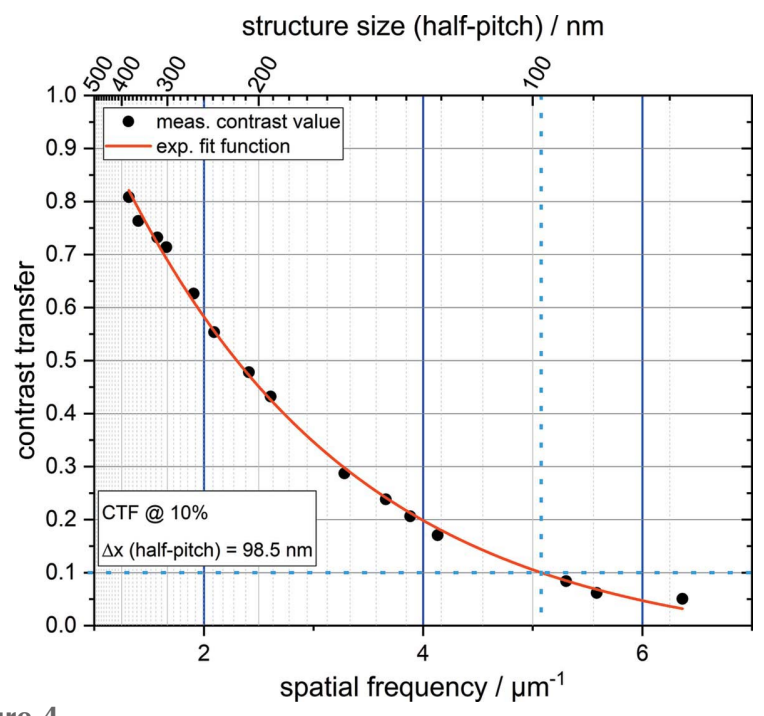

Figure 4

Contrast transfer function (CTF) calculated with the Siemens star pattern from Fig. 3. The spatial frequency for which the CTF reaches 0.1 corresponds to the Rayleigh resolution limit and has been determined through the exponential fit function shown in the graph.

star image of Fig. 3 was used to calculate the contrast for different radial positions along the spherical pattern. Each radius corresponds to a certain spatial frequency and the contrast transfer decreases as the spatial frequency increases. In Fig. 4 the resulting CTF is presented. According to Smith (1997), a decrease in the contrast transfer to $10 \%$ corresponds to the Rayleigh resolution limit, i.e. the minimum distance between two object points for which they can still be distinguished in the corresponding image. In our case, the CTF reaches $10 \%$ at a spatial frequency of $5.1 \mu^{-1}$, which corresponds to a half-pitch resolution of $98.5 \mathrm{~nm}$. This result is in accordance with the visual perception of the pattern, where the smallest structures with sizes $<100 \mathrm{~nm}$ are still discernible.

\subsection{XRF: four-channel SDD}

The four-channel SDD consists of four independent kidneyshaped SDD cells arranged like a cloverleaf around a central pinhole through which the excitation beam is guided to the sample (Fig. 2, right). Each SDD cell has an active area of about $15 \mathrm{~mm}^{2}$, resulting in an overall active area of $60 \mathrm{~mm}^{2}$.

Excitation radiation passes the central pinhole on the way to the sample and fluorescence radiation in backscatter geometry (Fig. 2, left) is detected on each of the four individual SDD cells. This geometry, in combination with the central pinhole and flat design, allows the detector to be positioned close to the sample and thus reach a very high solid angle of detection.

To shield the silicon chip from photoelectrons, it is equipped with a $0.5 \mu \mathrm{m}$-thick Mylar window. Due to this comparably thin window, the threshold energy of the four-channel SDD is reduced to $140 \mathrm{eV}$, assuming $\sim 15 \%$ transmission through the Mylar window for a mean incidence angle of $45.5^{\circ}$ at the optimum distance from the sample. Electrons up to $\sim 3 \mathrm{keV}$ are trapped in the Mylar window.
In contrast to the $\mathrm{CCD}$, the readout time of the four-channel SDD is not the limiting factor. In theory, readout times can be set close to the response time $(\sim 1-2 \mu \mathrm{s})$, but would be too short to gather meaningful XRF spectra. Estimations of reasonable minimal acquisition times can be carried out based on the maximum throughput of the four-channel SDD. Assuming fluorescence intensities close to the maximum throughput $\left(\sim 2 \times 10^{6}\right.$ photons $\mathrm{s}^{-1}$, according to the manufacturer) and at least 500-1000 events in the XRF spectrum, the minimum acquisition time would be $0.5 \mathrm{~ms}$. This is in the same range as the acquisition time of the transmission detection system consisting of the CCD ( $2 \mathrm{~ms}$ without binning) and a phosphor screen (1 ms decay time). However, for real applications with sample inhomogeneities, acquisition times of at least $5 \mathrm{~ms}$ are necessary. For quantitative evaluation where elemental maps strongly depend on good counting statistics, even higher acquisition times are recommended.

In order to facilitate short measurement times, the fourchannel SDD is operated in live-stream mode, where each event of the multi-channel analyser is transferred to the PC according to the sequence of the incoming photons. During the measurement, this live-stream is transformed into a spectrum for each measurement point, so that pre-selected regions of interest (ROIs) within these spectra can be summed. The ROIs, i.e. the elements of interest, are plotted once a scanned line is completed. Thus, a special map for each selected element is obtained, showing its occurrence and distribution within the sample.

A more precise spectrum evaluation requires a deconvolution of the characteristic lines, background stripping and consideration of detector effects (escape peaks, pile-up, sensitivity etc.) and has to be carried out after the experiments. Well known experimental conditions, combined with a careful fitting of the spectra and using the fundamental parameter (FP) method, will in principle enable quantitative imaging. However, absorption effects of the fluorescence radiation for large solid angles of detection in inhomogeneous samples need to be taken into account and present a challenge for real quantification.

2.2.1. Large solid angle of detection - optimum detector position. Due to its ring-like shape (see Fig. 2, right), the solid angle of detection as a function of distance $d$ to the sample can be determined using the formula for circular detectors (Zaluzec, 2014). For a ring, the solid angle is simply the difference between the solid angle for full circles of the outer radius $r_{\mathrm{o}}$ and the inner radius $r_{\mathrm{i}}$,

$$
\begin{aligned}
\Omega(d)= & 2 \pi\left(1-f_{\mathrm{s}}\right) \\
& \times\left[\frac{r_{\mathrm{o}}^{2}+d^{2}-d\left(r_{\mathrm{o}}^{2}+d^{2}\right)^{1 / 2}}{r_{\mathrm{o}}^{2}+d^{2}}-\frac{r_{\mathrm{i}}^{2}+d^{2}-d\left(r_{\mathrm{i}}^{2}+d^{2}\right)^{1 / 2}}{r_{\mathrm{i}}^{2}+d^{2}}\right]
\end{aligned}
$$

The factor $f_{\mathrm{s}} \in[0 ; 1]$ describes the reduction of the active area by obstacles in the beam path, e.g. stabilization grids on the detector surface. 
Table 1

Elemental composition of the XRF calibration sample.

Mass depositions are average values measured independently by GAAS, FAAS, ICP-OES and TXRF. Not all fluorescence lines listed here can be found in the XRF spectrum due to very low cross sections and absorption in the $0.5 \mu \mathrm{m}$ Mylar window in front of the four-channel SDD. Fluorescence energies below the cut-off energy of $\sim 140 \mathrm{eV}$ of the system are not listed.

\begin{tabular}{lcl}
\hline $\begin{array}{l}\text { Element/ } \\
\text { composition }\end{array}$ & $\begin{array}{l}\text { Mass } \\
\text { deposition } \\
\left(\mathrm{ng} \mathrm{mm}^{-2}\right)\end{array}$ & $\begin{array}{l}\text { Significant fluorescence lines with corresponding } \\
\text { absorption edges excited by } 1 \mathrm{keV}(\mathrm{eV}) \dagger\end{array}$ \\
\hline $\mathrm{Pb}$ & $84.9 \pm 12.3$ & \\
$\mathrm{La}$ & $121.4 \pm 14.5$ & $M 5-N 6,7(836) ; M 4-N 6(853) ; M 4,5-N 6,7(647.2)$ \\
$\mathrm{Pd}$ & $23.3 \pm 4.5$ & $M 4,5-N 2,3(284.8)$ \\
$\mathrm{Mo}$ & $8.6 \pm 0.9$ & $M 4,5-N 2,3(193.5)$ \\
$\mathrm{Cu}$ & $22.2 \pm 3.3$ & $L 3-M 5(927.7) ; L 2-M 4(947.3)$ \\
$\mathrm{Fe}$ & $433.9 \pm 5.9$ & $L 3-M 5(704.8) ; L 2-M 4(717.9)$ \\
$\mathrm{Si}_{3} \mathrm{~N}_{4}$ & Substrate & Si: $L 1-M 2(147.7)$ \\
& & N: $K-L 2(392.4) ; K-L 1(372.6)$ \\
\hline
\end{tabular}

$\dagger$ IUPAC notation; energies from the ELAM database (Elam et al., 2002).

This function has a global maximum of $\Omega(d)=1.1$ sterad at a distance of $d_{\mathrm{opt}}=2.42 \mathrm{~mm}$, where $d_{\mathrm{opt}}$ is the distance of the mounting of the four-channel SDD (not the active crystals) to the sample surface. Due to the very tight geometry of the setup, the distance to the active crystals of the four-channel SDD cannot be estimated during the alignment procedure. The value of $d_{\mathrm{opt}}$ varies slightly in an interval of $\pm \Delta d$ around the calculated value, so that in the range between $d_{\min }=$ $1.81 \mathrm{~mm}$ and $d_{\min }=3.28 \mathrm{~mm}$ the solid angle of detection still exceeds $95 \%[\Omega(d)=1.05$ sterad]. It is therefore favourable to position the detector within this distance interval to the sample surface.

To verify the assumptions resulting from these calculations, a homogeneous sample (Table 1) was measured with an excitation energy of $1.5 \mathrm{keV}$ and XRF spectra were collected for different sample-to-detector distances. The results are presented in Fig. 5, where the intensity in counts per second (in the energy range $0.253-1.341 \mathrm{keV}$ ) has been plotted as a function of the distance between the sample surface and the mounting of the four-channel SDD. The fitted curve (grey) was obtained by adapting the distance-offset mentioned above

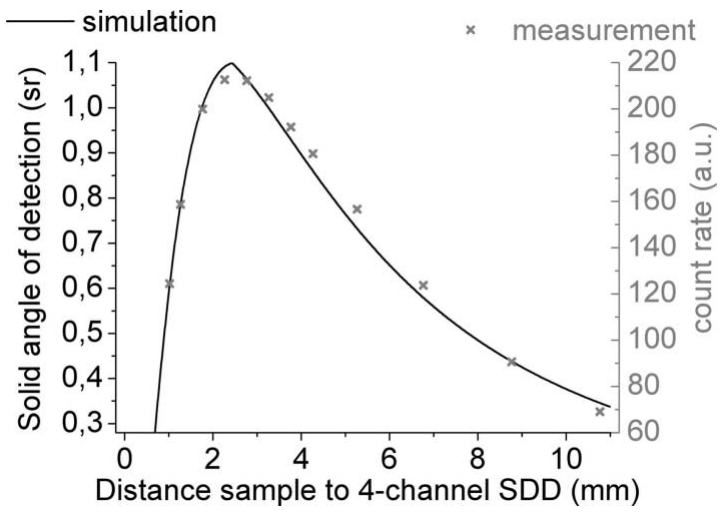

Figure 5

Distance dependance (four-channel SDD to sample) of measured and simulated intensities. and the maximum solid angle of detection was correlated to the maximum detected intensity.

2.2.2. Large solid angle of detection - absorption effects. A large solid angle of detection is directly associated with a variation of the observation angle. In the common approaches for XRF quantification relying on the Sherman equation, the entrance and exit beams are considered to be well collimated. In particular, the detector is assumed to subtend only a small solid angle of detection (Sherman, 1955). Of course, this assumption is not valid for solid angles of detection of up to 1.1 sterad.

Although the problem of divergent angles and explicitly large solid angles of detection has been researched with various approaches ever since Sherman published his article in 1955 (Bonizzoni et al., 2006; Chang \& Wittry, 1994; Malzer \& Kanngießer, 2003; Mantler \& Kawahara, 2004; Pavlinsky \& Kitov, 1979), no full analytical incorporation of divergent angles into the Sherman equation has yet been published or adopted by standard quantification algorithms. Nevertheless, for homogeneous samples, the equivalent angle approach, like the equivalent angle model by Malzer \& Kanngießer (2003), is applicable for our STXM-XRF experiments.

In cases of inhomogeneous samples, where the fluorescence radiation is not only attenuated by different pathways through the sample but also by different matrices, approaches like the equivalent angle model by Malzer \& Kanngießer (2003) become obsolete. Here, a reliable quantification approach has yet to be developed.

\section{Experimental}

Experiments were primarily carried out at the variable polarization XUV beamline P04 of PETRA III (Viefhaus et al., 2013). P04 is currently one of the world's most brilliant beamlines in the soft and tender X-ray range; the source brilliance is $10^{20}$ photons $\mathrm{s}^{-1} \mathrm{~mm}^{-2} \mathrm{mrad}^{-2}(0.1 \%$ bandwidth $)^{-1}(100 \mathrm{~mA})^{-1}$ and the resolving power exceeds 10000 for the whole energy range $250-3000 \mathrm{eV}$. For our demands, a quasi-parallel beam with $5 \mathrm{~mm}(\mathrm{~h}) \times 2 \mathrm{~mm}(\mathrm{v})$ beam size on the $\mathrm{ZP}$ was chosen.

The energy range which can currently be realized with the STXM-XRF setup ranges from $700 \mathrm{eV}$ to $3000 \mathrm{eV}$. Since the four-channel SDD is placed between the sample and the zone plate, a further reduction of the sample-ZP distance, in order to access lower energies, is not possible. Therefore, energies lower than $700 \mathrm{eV}$ are not accessible with the current ZP. A different type of focusing ZP can enlarge the energy range down to the limit of the beamline, which is currently marginally lower than the $\mathrm{C} K$-edge at $\sim 250 \mathrm{eV}$.

The flux on the sample was not measured directly due to the lack of a calibrated diode. Nevertheless, with the help of a well documented XRF calibration sample (Hönicke et al., 2018), assuming a maximum solid angle of detection of $1.1 \mathrm{sterad}$, the photon flux on the sample is estimated to be $I_{0} \simeq 3 \times$ $10^{9}$ photons s $^{-1}$. Considering the ratio of the beam area to the active area of the $\mathrm{ZP}$ and the transmission of the $\mathrm{ZP}$, this flux is in the expected range. 
Table 2

Scan parameters at different spots on the XRF calibration sample.

\begin{tabular}{lcc}
\hline $\begin{array}{l}\text { Number of measurement } \\
\text { points }(\mathrm{h} \times \mathrm{v})\end{array}$ & $\begin{array}{l}\text { Step width } \\
(\mathrm{nm})\end{array}$ & $\begin{array}{l}\text { Acquisition time } \\
(\mathrm{ms})\end{array}$ \\
\hline $100 \times 100$ & 1000 & 5 \\
$100 \times 100$ & 1000 & 10 \\
$100 \times 100$ & 1000 & 15 \\
$200 \times 200$ & 500 & 10 \\
$200 \times 200$ & 100 & 20 \\
\hline
\end{tabular}

\subsection{Limit of detection}

A laterally homogeneous XRF calibration sample (Hönicke et al., 2018) was used to estimate the limit of detection (LOD) for $\mathrm{La}, \mathrm{Cu}$ and $\mathrm{Fe}$. The XRF calibration sample consists of thin deposition layers of these elements on a commercially available silicon nitride membrane. The layer sequence, the average mass depositions and the fluorescence lines with their corresponding absorption edges for an excitation energy of $1 \mathrm{keV}$ are listed in Table 1. To increase stability, XRF calibration samples are always covered with a thin $\mathrm{C}$ layer, but, since no mass deposition for $\mathrm{C}$ was determined, it is not listed in this table.

We recorded several lateral maps with varying acquisition times and step widths, as presented in Table 2. For $5 \mathrm{~ms}, 10 \mathrm{~ms}$ and $20 \mathrm{~ms}$, single XRF spectra were used to estimate the LOD and were summed as 'long-time spectra'. For example, 25 spectra from different positions with $20 \mathrm{~ms}$ acquisition times were summed in order to obtain the XRF spectrum for $500 \mathrm{~ms}$. Our estimation of the LODs is based on the common definition (Streli et al., 2006; Rousseau, 2001)

$$
\mathrm{LOD}=X_{\mathrm{mcQ}}\left\{\frac{3\left[\left(I_{\mathrm{R}}-I_{\mathrm{N}}\right) / t\right]^{1 / 2}}{I_{\mathrm{N}}}\right\}
$$

where $I_{\mathrm{R}}$ represents the raw peak intensity, $I_{\mathrm{N}}$ is the net peak intensity and $t$ is the acquisition time. The expression $X_{\mathrm{mcQ}}$ corresponds to the mass, the concentration or the mass deposition, depending on the method.

However, it is important to keep in mind that LODs only provide a rough estimation of detectable traces in samples. For strong overlapping fluorescence lines, this procedure cannot be applied.

The intensities $I_{\mathrm{R}}$ and $I_{\mathrm{N}}$ were determined by using the ROI procedure of PyMCA (Solé et al., 2007). As shown in Table 1 and Fig. 6, the elements $\mathrm{La}, \mathrm{Cu}$ and Fe generate intense $L$ - or $M$-fluorescence lines for an excitation energy of $1 \mathrm{keV}$. Fig. 6 shows XRF spectra for $5 \mathrm{~ms}, 50 \mathrm{~ms}$ and $500 \mathrm{~ms}$. The peaks are assigned to the respective elements. The additional elements, which are not mentioned in Table 1 , are $\mathrm{C}$ (stabilization layer) and $\mathrm{O}$. The latter originates from the Fe layer, since iron oxide was used as the target material for the Fe deposition layer.

The estimated LODs are presented in Table 3. The combination of the high-brilliance $\mathrm{P} 04$ beamline and the large solid angle of detection yields LODs in the range of $1 \mathrm{ng} \mathrm{mm}^{-2}$ for an acquisition time of $20 \mathrm{~ms}$ for $\mathrm{Cu}, 50 \mathrm{~ms}$ for $\mathrm{Fe}$ and $500 \mathrm{~ms}$ for La. Due to the reduced photoproduction
Table 3

$\mathrm{LOD}$ for $\mathrm{La}, \mathrm{Cu}$ and $\mathrm{Fe}$ for acquisition times varying from $5 \mathrm{~ms}$ to $800 \mathrm{~s}$ and an excitation energy of $1 \mathrm{keV}$.

Missing values are due to little statistics and a calculation of the background intensity of zero $\left(I_{\mathrm{R}}-I_{\mathrm{N}}=0\right)$.

\begin{tabular}{lrrr}
\hline $\begin{array}{l}\text { Acquisition time } \\
(\mathrm{ms})\end{array}$ & \multicolumn{1}{c}{$\begin{array}{c}\mathrm{LOD}_{\mathrm{La}} \\
\left(\mathrm{ng} \mathrm{mm}^{-2}\right)\end{array}$} & \multicolumn{1}{c}{$\begin{array}{l}\mathrm{LOD}_{\mathrm{Cu}} \\
\left(\mathrm{ng} \mathrm{mm}^{-2}\right)\end{array}$} & \multicolumn{1}{c}{$\begin{array}{l}\mathrm{LOD}_{\mathrm{Fe}} \\
\left(\mathrm{ng} \mathrm{mm}^{-2}\right)\end{array}$} \\
\hline 5 & & $10.42 \pm 1.55$ & $22.09 \pm 2.96$ \\
20 & $25.59 \pm 3.05$ & $0.69 \pm 0.10$ & $2.00 \pm 0.27$ \\
50 & $6.30 \pm 0.75$ & $0.41 \pm 0.06$ & $0.64 \pm 0.09$ \\
500 & $0.83 \pm 0.10$ & $0.07 \pm 0.01$ & $0.12 \pm 0.02$ \\
10000 & $0.05 \pm 0.01$ & $<0.01$ & $0.01 \pm<0.01$ \\
100000 & $<0.01$ & $<0.01$ & $<0.01$ \\
\hline
\end{tabular}

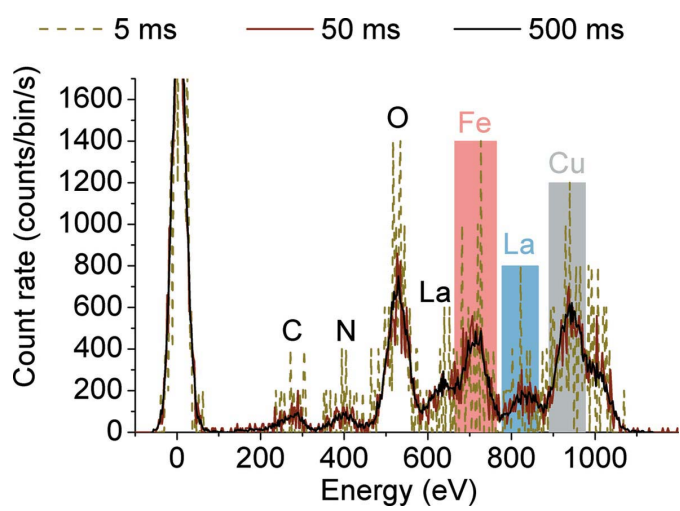

Figure 6

XRF spectra for $5 \mathrm{~ms}, 50 \mathrm{~ms}$ and $500 \mathrm{~ms}$. ROIs used for the calculation of LOD are highlighted in red $(\mathrm{Fe})$, blue $(\mathrm{La})$ and grey $(\mathrm{Cu})$.

cross section of the $M$-fluorescence, the acquisition time for $\mathrm{La}$ must be higher to obtain the same LOD. The excitation energy of $1 \mathrm{keV}$ is very close to the $\mathrm{Cu} L_{\mathrm{III} / \mathrm{II}^{-}}-$edge and thus $\mathrm{Cu}$ is the most sensitive element from this selection.

The high sensitivity for light elements of the AnImaX setup enables the analysis of trace elements in all kinds of application areas like biomedical, environmental or food production research. The access of imaging light trace elements in reasonable time periods opens complete new application fields. In addition, the sensitivity of $L$ - and $M$-fluorescence allows the analysis of heavy trace elements at the same time. Previously, measurements had to be performed twice, at soft to tender and at hard beamlines, in order to access traces of light and heavy elements.

\subsection{Biomedical application}

Imaging is an important tool for biological and biomedical research and optical light microscopy is a well established method in laboratories all over the world. However, the spatial resolution of a microscope is limited by the wavelength of the probing radiation, which corresponds to a minimum of approximately $200 \mathrm{~nm}$ for visible-light microscopy (not taking into account novel super-resolution techniques). Therefore, methods with a higher spatial resolution are desirable. Even though electron microscopy can provide very high spatial resolutions in the Angstrom range, depth information can only 
be gained at the price of extensive sample preparation. X-ray microscopy, owing to its relatively high penetration depth, is a promising approach here, especially when combined with fluorescence detection.

3.2.1. Biomedical application - proof of principle. The biochemical mechanisms governing the transport of lipids from blood vessels across the endothelial layer into parenchymal cells of the brown adipose tissue (BAT) of mammals are not yet understood in detail. Better insights into the involved mechanisms of transendothelial lipid transport are mandatory to develop novel therapeutic strategies for the treatment of hyperlipidemia and metabolic diseases such as diabetes mellitus type or non-alcoholic steatohepatitis (Scheja \& Heeren, 2016).

In this study, we present the first proof-of-principle X-ray microscopy measurements on mice tissue samples. For this purpose, C57BL/6J mice receive an injection of lipoproteins labelled with superparamagnetic iron oxide (SPIO-lipoproteins) nanoparticles (Bruns et al., 2009). To compare conditions of low and high organ-specific SPIO-lipoprotein uptake, mice were housed at ambient room temperature (low lipid uptake activity into thermogenic BAT) or at $6^{\circ} \mathrm{C}$ to activate thermogenesis. The cold activation stimulates an enhanced lipid uptake into BAT (Bartelt et al., 2011; Schlein et al., 2016; Heine et al., 2018). Hence, a higher concentration of SPIOlipoproteins, and thus higher Fe fluorescence in the BAT of cold-exposed mice in comparison with the control group, is expected.

The tissue samples of both groups were cut into slices of 4-8 $\mu \mathrm{m}$ thickness, fixated on a $\mathrm{Si}_{3} \mathrm{~N}_{4}$ window (100 nm thickness) with $2.5 \%$ formaldehyde and air dried afterwards. For the investigation, an excitation energy of $1 \mathrm{keV}$ was chosen, which is above and close to the Fe $L_{\mathrm{I}}$-edge $(0.8446 \mathrm{keV})$. The nominal SPIO particle size was specified to be $\sim 10 \mathrm{~nm}$, but agglomerations of the attached lipids were expected.

The tissue was scanned with a $100 \mathrm{~nm}$ step size and a $50 \mathrm{~ms}$ acquisition time in a range of $400 \times 400$ pixels. The resulting maps are shown in Figs. 7 and 8 at the bottom, where the detected $\mathrm{C}$ intensity is depicted in blue and the red represents the occurrence of Fe. At the top of Figs. 7 and 8, a transmission image of the exact same area is presented, so that structural and elemental information can be correlated. Since we know that brown adipocytes in BAT contains more $\mathrm{C}$ than blood vessels, we can conclude that the areas where high concentrations of $\mathrm{C}$ are detected correspond to adipocytes. Furthermore, as the natural concentration of Fe in organic tissue lies below the detection limit, the detected Fe fluorescence must correspond to the occurrence of SPIO-lipoproteins.

In Fig. 7, the recorded image presents some scanning artefacts as the same line was scanned several times due to a malfunction of the scanner controller. The repeated lines were cut from the image, resulting in a visible horizontal line. Furthermore, the storage ring changed from top-up to decay mode, causing a decrease in fluorescence and transmission intensity, visible in the bottom part of the image.

For the cold activated tissue in Fig. 8, the area in the black rectangle was scanned three times; each scan lasted for $50 \mathrm{~ms}$.
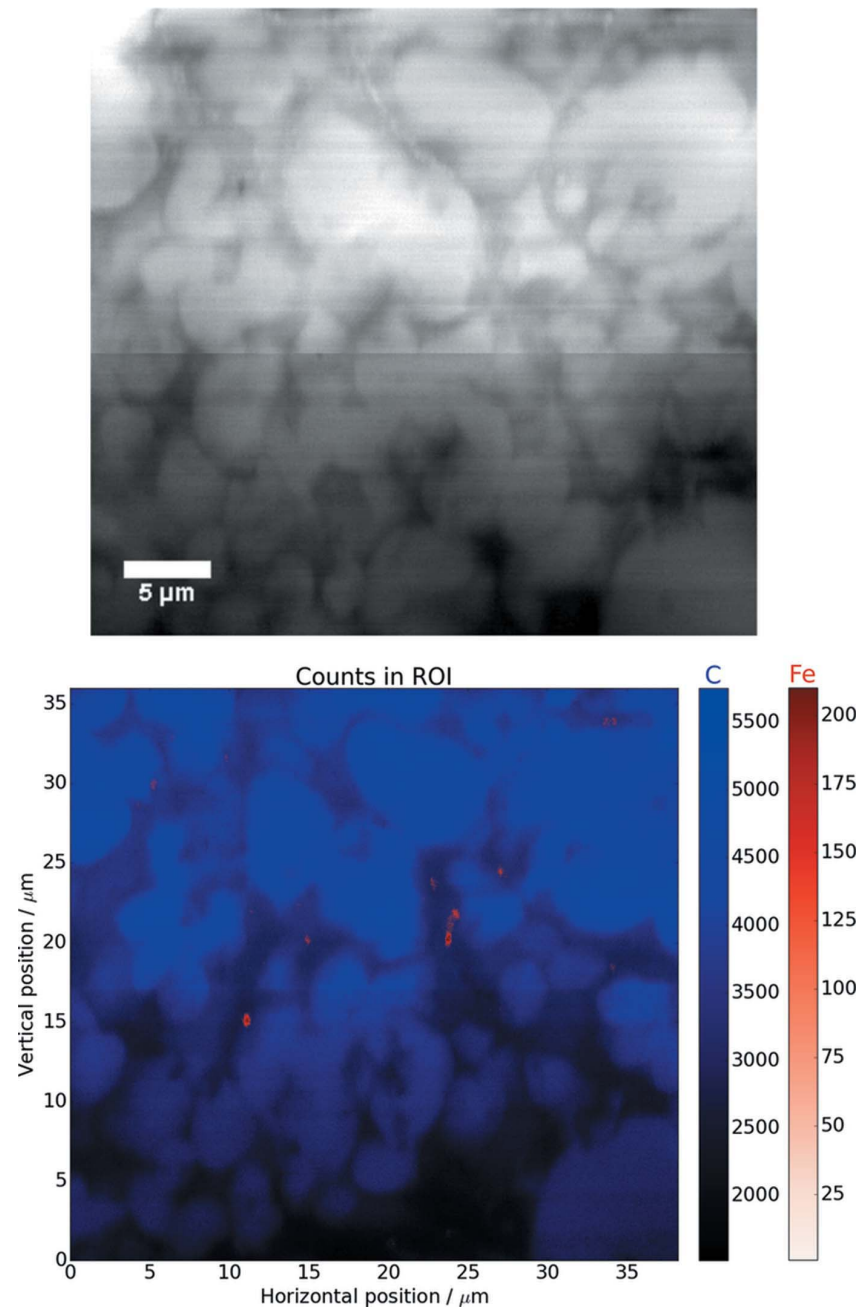

Figure 7

Transmission image (top) and pseudo-colour image (bottom) for C (blue, BAT) and Fe (red, SPIO) for control tissue.

In the transmission image this area appears much brighter than the rest, indicating radiation damage.

For a well grounded study of this biomedical research question, further measurements with higher statistics need to be performed.

Nevertheless, this example demonstrates the potential and possibilities of the AnImaX endstation with a large solid angle of detection in combination with the high-brilliance beamline P04 at PETRA III. To the best of our knowledge, a $50 \mathrm{~ms}$ acquisition time per pixel for the imaging of biomedical applications in the soft and tender X-ray range has not yet been achieved elsewhere.

\subsection{Radiation damage - absorbed dose estimation}

As shown in the previous section, radiation damage in biological samples can already be observed for very short measurement times of $50-150 \mathrm{~ms}$ per pixel. A first indicator for the estimation of radiation damage is the absorbed dose $D$. Depending on the application, signs of radiation damage were observed for $D=10^{4} \mathrm{~Gy}$ for unfixed hydrated biological samples (Schneider, 1998), for $D=10^{5}$ Gy for ascidian blood 


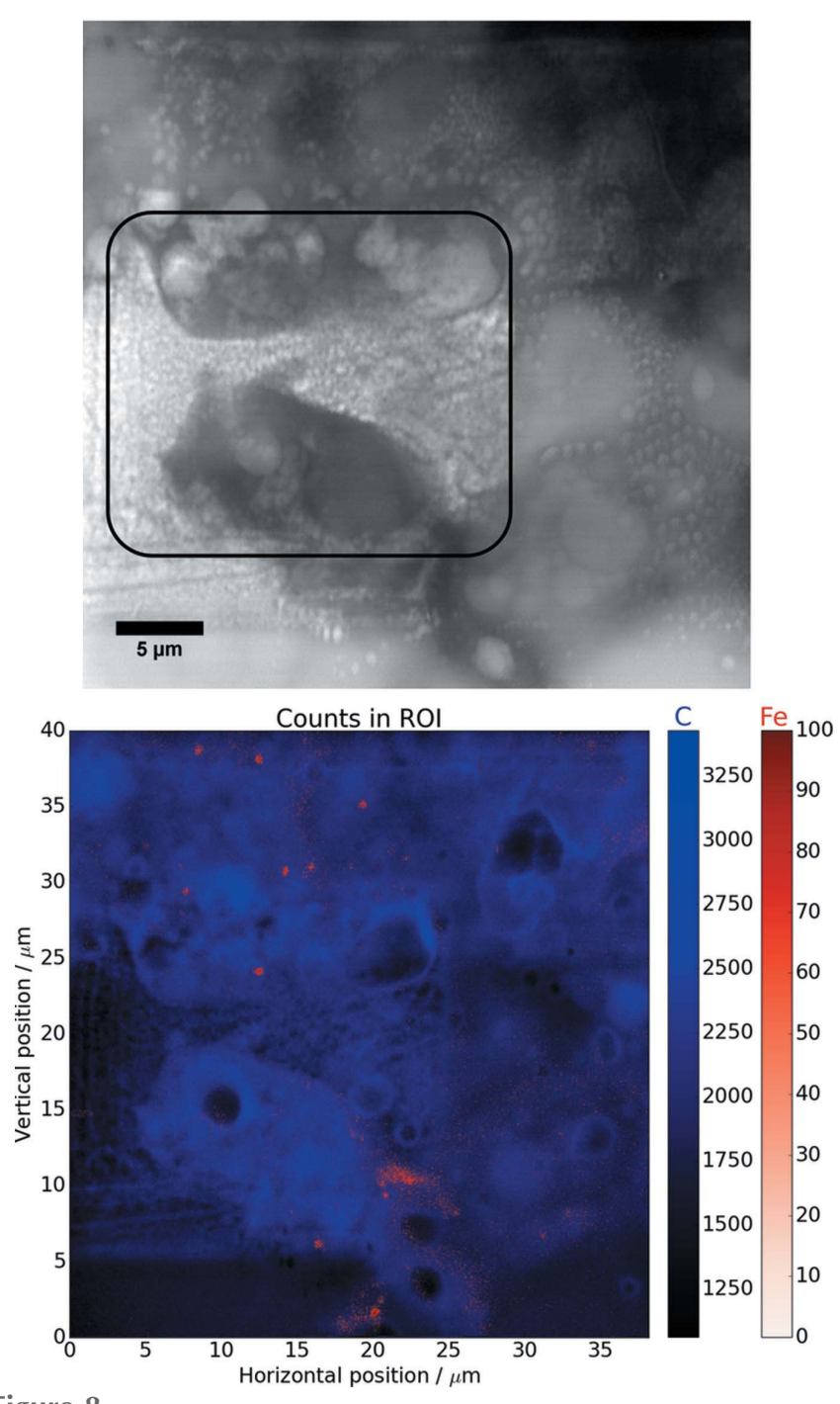

Figure 8

Transmission image (top) and pseudo-colour image (bottom) for C (blue, BAT) and Fe (red, SPIO) for cold-activated tissue. The box in the top image marks an area with radiation damage from previous scans (see text).

cells (Fayard et al., 2009) and $D \simeq 10^{7}$ Gy for formalin-fixed cells (Gianoncelli et al., 2015). The absorbed dose is defined as the ratio of the absorbed energy $\Delta E=I_{\mathrm{A}} h v$ and the mass $m$,

$$
D=\Delta E / m
$$

where $I_{\mathrm{A}}$ is the number of absorbed photons and $h v$ is the photon energy. $I_{\mathrm{A}}$ can be calculated based on the chemical composition, the density $\rho$ and the thickness $d$ of the sample. The mass can be estimated by assuming a rectangular illumination (volume $\simeq 100 \mathrm{~nm} \times 100 \mathrm{~nm} \times d$ ) and the sample density $\rho$.

Table 4 shows the empirical formulae and densities used for the dose estimation as well as the results of the calculated absorbed doses for $5 \mu \mathrm{m}$ - and $10 \mu \mathrm{m}$-thick samples and $100 \mathrm{~ms}$ acquisition time. The results in Table 4 demonstrate that, for all examples, the absorbed dose lies in the range between $5.5 \times 10^{5} \mathrm{~Gy}$ and $9.5 \times 10^{5} \mathrm{~Gy}$. As mentioned above, these are values for which radiation damage would already occur.
Table 4

Absorbed dose estimation for some applications in life science for $5 \mu \mathrm{m}$ and $10 \mu \mathrm{m}$-thick samples, an excitation energy of $1000 \mathrm{eV}$ at P04 and a $100 \mathrm{~ms}$ acquisition time.

\begin{tabular}{lllll}
\hline & & & $\begin{array}{l}\text { Dose for } \\
d=5 \mu \mathrm{m},\end{array}$ & $\begin{array}{l}\text { Dose for } \\
d=10 \mu \mathrm{m}\end{array}$ \\
Application & $\begin{array}{l}\text { Elemental } \\
\text { formula }\end{array}$ & $\begin{array}{l}\text { Density } \\
\left(\mathrm{g} \mathrm{cm}^{-3}\right)\end{array}$ & $\begin{array}{l}t=100 \mathrm{~ms} \\
\left(\times 10^{5} \mathrm{~Gy}\right)\end{array}$ & $\begin{array}{l}t=0 \mathrm{~ms} \\
\left(\times 10^{5} \mathrm{~Gy}\right)\end{array}$ \\
\hline Adipose & $\mathrm{C}_{57} \mathrm{H}_{104} \mathrm{O}_{6}$ & 0.94 & 6.6 & 8.9 \\
Bone & $\mathrm{O}_{1702} \mathrm{H}_{429} \mathrm{P}_{372} \mathrm{Ca}_{620} \mathrm{~N}_{34} \mathrm{C}_{134}$ & 1.70 & 5.5 & 5.6 \\
Water & $\mathrm{H}_{2} \mathrm{O}$ & 1.00 & 8.4 & 9.4 \\
Proteine & $\mathrm{C}_{94} \mathrm{H}_{139} \mathrm{~N}_{24} \mathrm{O}_{31} \mathrm{~S}$ & 1.35 & 6.1 & 7.0 \\
\hline
\end{tabular}

These considerations show the necessity of a cryogenic atmosphere for applications in life science. Under cryogenic conditions, radiation damage is strongly reduced and would only occur for absorbed doses in the range $10^{8}-10^{9} \mathrm{~Gy}$ (Schneider, 1998). Hence, a cryogenic atmosphere would allow much better statistics and is therefore mandatory for quantitative analysis.

\section{Conclusions}

The AnImaX endstation is a flexible X-ray microscope, which can be operated in full-field as well as in scanning mode. Since we have enabled on-the-fly mapping with acquisition times of a few milliseconds per pixel for the scanning mode, very timeefficient mappings of dose-sensitive samples are achievable, which is of great advantage for the investigation of biological samples. Furthermore, an energy-dispersive detector (fourchannel SDD) provides additional elemental information.

The implementation of the four-channel SDD detector, which was specially adapted for our setup, has been an important step in delivering correlated imaging and investigating the connection between structural and functional information in biological samples. With its large solid angle of detection of up to 1.1 sterad, acquisition times of less than $100 \mathrm{~ms}$ have become possible. With the help of well defined reference samples, it was possible to specify the LOD for La, $\mathrm{Cu}$ and $\mathrm{Fe}$ for different acquisition times. As a result, we could determine that an LOD of $1 \mathrm{ng} \mathrm{mm}^{-2}$ requires acquisition times of $500 \mathrm{~ms}$ for $\mathrm{La}, 20 \mathrm{~ms}$ for $\mathrm{Cu}$ and $50 \mathrm{~ms}$ for Fe. This example demonstrates that with this setup, in combination with a highly brilliant source, even trace elements with fluorescence lines of very low sensitivity, such as $M$-lines, can be detected.

The potential of AnImaX for biomedical applications in life science was demonstrated with the proof-of-principle measurements of SPIO-lipoprotein enriched BAT of coldactivated versus control mice. We were able to measure trace elements in these biomatrices with very short acquisition times of $50 \mathrm{~ms}$, which is unprecedented in this energy range. Thus, AnImaX opens possibilities for various research fields such as the investigation of the toxicity of nanoparticles, the development of drug carriers or the processes of food production.

Nevertheless, even these short measurement times caused radiation damage. Hence, AnImaX will soon be equipped with a cryo-tomography station. A cryogenic environment reduces 
radiation damage significantly and the rotation stage will allow us to perform FF-XRM tomography as a first step, followed by experiments to establish tomographic measurements in STXM mode. Longer measurement times facilitated by the cryogenic environment will also help to further develop our quantitative approach for large solid angles of detection.

In order to enlarge the research fields of the AnImaX setup even more, the energy range below $700 \mathrm{eV}$ needs to be accessible. Different approaches like varying types of focusing ZPs or another mounting point of the OSA will be tested.

\section{Funding information}

We acknowledge the BMBF for funding the AnImaX joint project (grant No. 05 K2016) and the previous joint project (FlexIX - Flexible Imaging with X-rays; grant No. 05 K2013) project. We also thank AXO DRESDEN GmbH for providing the XRF calibration sample.

\section{References}

Bartelt, A., Bruns, O. T., Reimer, R., Hohenberg, H., Ittrich, H., Peldschus, K., Kaul, M. G., Tromsdorf, U. I., Weller, H., Waurisch, C., Eychmüller, A., Gordts, P. L. S. M., Rinninger, F., Bruegelmann, K., Freund, B., Nielsen, P., Merkel, M. \& Heeren, J. (2011). Nat. Med. 17, 200-205.

Bonizzoni, L., Maloni, A. \& Milazzo, M. (2006). X-ray Spectrom. 35, 390-399.

Bruns, O. T., Ittrich, H., Peldschus, K., Kaul, M. G., Tromsdorf, U. I., Lauterwasser, J., Nikolic, M. S., Mollwitz, B., Merkel, M., Bigall, N. C., Sapra, S., Reimer, R., Hohenberg, H., Weller, H., Eychmüller, A., Adam, G., Beisiegel, U. \& Heeren, J. (2009). Nat. Nanotechnol. 4, 193-201.

Bufon, J., Schillani, S., Altissimo, M., Bellutti, P., Bertuccio, G., Billè, F., Borghes, R., Borghi, G., Cautero, G., Cirrincione, D., Fabiani, S., Ficorella, F., Gandola, M., Gianoncelli, A., Giuressi, D., Kourousias, G., Mele, F., Menk, R. H., Picciotto, A., Rachevski, A., Rashevskaya, I., Sammartini, M., Stolfa, A., Zampa, G., Zampa, N., Zorzi, N. \& Vacchi, A. (2018). J. Instrum. 13, C03032.

Chang, W. Z. \& Wittry, D. B. (1994). Microbeam Anal. 3, 23-32.

Eichert, D. (2015). X-ray Microscopy. In Synchrotron Radiation, edited by Mobilio S., Boscherini F., Meneghini C. Berlin/ Heidelberg: Springer.

Elam, W. T., Ravel, B. D. \& Sieber, J. R. (2002). Radiat. Phys. Chem. 63, 121-128.

Fayard, B., Salomé, M., Takemoto, K., Kihara, H. \& Susini, J. (2009). J. Electron Spectrosc. Relat. Phenom. 170, 19-24.

Gianoncelli, A., Bufon, J., Ahangarianabhari, M., Altissimo, M., Bellutti, P., Bertuccio, G., Borghes, R., Carrato, S., Cautero, G., Fabiani, S., Giacomini, G., Giuressi, D., Kourousias, G., Menk,
R. H., Picciotto, A., Piemonte, C., Rachevski, A., Rashevskaya, I., Stolfa, A., Vacchi, A., Zampa, G., Zampa, N. \& Zorzi, N. (2016). Nucl. Instrum. Methods Phys. Res. A, 816, 113-118.

Gianoncelli, A., Vaccari, L., Kourousias, G., Cassese, D., Bedolla, D. E., Kenig, S., Storici, P., Lazzarino, M. \& Kiskinova, M. (2015). Sci. Rep. 5, 10250.

Heeren, J. \& Scheja, L. (2018). Curr. Opin. Lipidol. 29, 180185.

Heine, M., Fischer, A. W., Schlein, C., Jung, C., Straub, L. G., Gottschling, K., Mangels, N., Yuan, Y., Nilsson, S. K., Liebscher, G., Chen, O., Schreiber, R., Zechner, R., Scheja, L. \& Heeren, J. (2018). Cell Metab. 28, 644-655.e4.

Holt, M., Harder, R., Winarski, R. \& Rose, V. (2013). Annu. Rev. Mater. Res. 43, 183-211.

Hönicke, P., Krämer, M., Lühl, L., Andrianov, K., Beckhoff, B., Dietsch, R., Holz, T., Kanngießer, B., Weißbach, D. \& Wilhein, T. (2018). At. Spectrosc. 145, 36-42.

Hornberger, B., de Jonge, M. D., Feser, M., Holl, P., Holzner, C., Jacobsen, C., Legnini, D., Paterson, D., Rehak, P., Strüder, L. \& Vogt, S. (2008). J. Synchrotron Rad. 15, 355-362.

Kaulich, B., Susini, J., David, C., Di Fabrizio, E., Morrison, G. R., Thieme, J., Wilhein, T., Kovac, J., Bacescu, D., Salome, M. \& others (2003). Synchrotron Radiation News. 16(3), 49-52.

Lider, V. V. (2017). Phys. Usp. 60, 187-203.

Malzer, W. \& Kanngießer, B. (2003). X-ray Spectrom. 32, 106-112.

Mantler, M. \& Kawahara, N. (2004). Rigaku J. 21, 17-25.

Obst, M. \& Schmid, G. (2014). 3D Chemical Mapping: Application of Scanning Transmission (Soft) X-ray Microscopy (STXM) in Combination with Angle-Scan Tomography in Bio-, Geo-, and Environmental Sciences. In Electron Microscopy: Methods and Protocols, edited by John Kuo. New York: Humana Press.

Pavlinsky, G. V. \& Kitov, B. I. (1979). X-ray Spectrom. 8, 96-101.

Rousseau, R. M. (2001). Rigaku J. 18, 33-47.

Scheja, L. \& Heeren, J. (2016). J. Hepatol. 64, 1176-1186.

Schlein, C., Talukdar, S., Heine, M., Fischer, A. W., Krott, L. M., Nilsson, S. K., Brenner, M. B., Heeren, J. \& Scheja, L. (2016). Cell Metab. 23, 441-453.

Schneider, G. (1998). Ultramicroscopy, 75, 85-104.

Sherman, J. (1955). Spectrochim. Acta, 7, 283-306.

Smith, S. W. (1997). The Scientist and Engineer's Guide to Digital Signal Processing. San Diego, CA: California Technical Publishing.

Solé, V. A., Papillon, E., Cotte, M., Walter, Ph. \& Susini, J. (2007). At. Spectrosc. 62, 63-68.

Streli, C., Wobrauschek, P., Fabry, L., Pahlke, S., Comin, F., Barrett, R., Pianetta, P., Lüning, K. \& Beckhoff, B. (2006). Total-Reflection $X$-ray Fluorescence (TXRF) Wafer Analysis. In Handbook of Practical X-ray Fluorescence Analysis, edited by B. Beckhoff, B. Kanngieser, N. Langhoff, R. Wedell \& H. Wolff. Berlin/Heidelberg: Springer-Verlag

Viefhaus, J., Scholz, F., Deinert, S., Glaser, L., Ilchen, M., Seltmann, J., Walter, P. \& Siewert, F. (2013). Nucl. Instrum. \& Methods Phys. Res. $A, \mathbf{7 1 0}, 151-154$.

Zaluzec, N. J. (2014). Microsc. Microanal. 20, 1318-1326. 